\title{
Path-integral Monte Carlo simulations for interacting few-electron quantum dots with spin-orbit coupling
}

\author{
Stephan Weiss and R. Egger \\ Institut für Theoretische Physik, Heinrich Heine Universität, D-40225 Düsseldorf, Germany
}

(Dated: July 19, 2021)

\begin{abstract}
We develop path-integral Monte Carlo simulations for a parabolic two-dimensional (2D) quantum dot containing $N$ interacting electrons in the presence of Dresselhaus and/or Rashba spin-orbit couplings. Our method solves in a natural way the spin contamination problem and allows for numerically exact finite-temperature results at weak spin-orbit coupling. For $N<10$ electrons, we present data for the addition energy, the particle density, and the total spin $S$ in the Wigner molecule regime of strong Coulomb interactions. We identify magic numbers at $N=3$ and $N=7$ via a peak in the addition energy. These magic numbers differ both from weak-interaction and classical predictions, and are stable with respect to (weak) spin-orbit couplings.
\end{abstract}

PACS numbers: 73.21.La, 71.70.Ej, 73.20.Qt

\section{INTRODUCTION}

Interacting few-body quantum systems have attracted a lot of attention over the past decades. In that respect, quantum dots (QDs) continue to be of fundamental interest to several fields, e.g., spintronics, nanoelectronics, and quantum computing. QDs are small solid-state devices, typically containing a few up to several hundred electrons confined in all space directions 1, 2]. They can be fabricated and studied using different approaches and materials 3, 4, 5, 6, 7], and control over both the charge and the spin degree of freedom of the confined electrons has been reported in experiments. Here, we address QDs as realized in two-dimensional (2D) semiconductor devices, e.g., in ultraclean $2 \mathrm{D}$ electron gases. We consider the case of parabolically confined individual QDs, which is quite appropriate in most practical cases 11. QDs offer the possibility to tune Coulomb correlations among electrons via external gates.

Spin properties of quantum dots have recently entered the focus of research 8, 9, 10, 11, 12], in particular as they are central both to quantum computation 13 and to spintronics 14]. Here spin-orbit (SO) terms have to be taken into account, coupling the spin dynamics to the orbital motion. In general, SO coupling is a relativistic effect, and appears to second order in the fine structure constant. In most materials of interest, two main mechanisms may be distinguished, namely Rashba 15. and Dresselhaus [16] SO couplings. The Rashba SO coupling strength $\alpha_{R}$ is due to the surface inversion asymmetry present in the confinement to a $2 \mathrm{D}$ electron gas, and therefore can be tuned by external gates [8]. The Dresselhaus coupling $\alpha_{D}$ is generally not tunable but can be important if the host crystal has no bulk inversion symmetry, e.g., in zincblende semiconductors. For simplicity, we only consider the linear Dresselhaus term and neglect various additional spin-orbit contributions, see Ref. 17] for an extended discussion. These contributions can in principle be taken into account within our approach. Furthermore, we focus on the case of vanishing magnetic field.

In this work, we investigate the behavior of a closed parabolic few-electron QD in the presence of spin-orbit couplings, containing up to $N=9$ interacting electrons. While the numerically exact method employed here allows to study arbitrary interactions in principle, it is probably most useful in the regime of intermediate-tostrong Coulomb interactions, where a 'Wigner molecule' 18, 19, 20, 21, 22, 23, 24] is formed. In terms of the standard electron gas parameter $r_{s}$, the studied interactions are around $r_{s} \approx 5$ to 10 . Then a clear tendency towards Wigner crystallization can be observed, but different spatial "shells" are not yet locked relative to each other as happens for very large $r_{s}$ [20]. In fact, while for $r_{s} \rightarrow \infty$, a completely classical situation is encountered [25, 26], quantum effects still play a major role for the 'incipient' Wigner molecule of interest here. In such a case, many standard calculational tools, e.g., exact diagonalization [27], the Hartree-Fock approximation, the fixed-node 28] or variational [29] Monte Carlo approach, or density functional theory can meet various difficulties (like artificial symmetry breakings) or require explicit justification, see Ref. 1] for a review. In that situation, finite-temperature path-integral Monte Carlo (PIMC) simulations represent an attractive alternative scheme. The case of no spin-orbit coupling has been studied using PIMC in Refs. 18, 20, 21, 23.

Before describing our PIMC scheme and the ensuing results, let us first discuss previous theoretical approaches to the physics of QDs in the presence of $\mathrm{SO}$ couplings. To study their effect on single-particle energies, one may set up perturbation theory for small SO couplings. Due to the linear dependence on momentum in Eq. (2) below, perturbation theory starts at second order and gives a quadratic decrease of single-particle energies with increasing Rashba coupling $\alpha_{R}$ (or Dresselhaus coupling $\alpha_{D}$ ) 30. Single-particle energy level crossings induced by the SO coupling have been discussed in Refs. 17, 31, 32 as a function of an applied magnetic field. Here, we are mainly concerned with many-body effects due to the Coulomb interaction. For two electrons, 
exact diagonalization studies have been carried out for rather strong Rashba couplings and weak interactions, as appropriate for InSb dots 33, 34]. Energy spectra were examined, a jump in the magnetization of the dot as a function of magnetic field was found [33], and a favoring of exchange over direct interactions as a consequence of SO interactions was discussed [34]. Governale [35] has employed spin-density functional theory for $N \leq 16$. He found that a very strong SO coupling leads to new peaks and/or the suppression of Hund's rule peaks otherwise present in the addition energy spectrum. An additional in-plane magnetic field was argued to imply paramagnetic behavior. Finally, in Ref. [36], several approximate schemes have been employed to study SO effects in weakly interacting QDs with $N \approx 11$ to 13 electrons. Here we provide results for $N<10$ electrons with strong Coulomb interactions and SO couplings, where exact diagonalization techniques may not apply anymore. One should also note that for $r_{s} \approx 5$ to 10 , spin effects are very important but necessitate an essentially exact treatment. For a recent comparison of QMC data to density functional theory in that respect, see Ref. 37.

In the absence of spin-orbit couplings, PIMC simulations for QDs suffer from two well-known problems, namely the fermionic sign problem and the spin contamination problem [18]. (The first problem can be relieved to some extent by the multilevel blocking algorithm [19]. Here we restrict ourselves to a simpler "bruteforce" approach.) The spin contamination problem arises for $\alpha_{R}=\alpha_{D}=0$ because both the total QD spin $S$ and its $z$ component $S_{z}$ are good quantum numbers. Now $S_{z}$ is in practice fixed during the simulation since there are no spin flip terms in the hamiltonian. At finite temperature, one then arrives at the undesirable situation where states with different $S$ but the same $S_{z}$ contribute to the simulation. This considerably complicates data analysis and represents a well-known problem affecting also other schemes, e.g., Hartree-Fock calculations. In the presence of spin-orbit coupling, however, neither $S$ nor $S_{z}$ are good quantum numbers and the full space of all $\left\{S_{z}, S\right\}$ becomes accessible. With increasing SO couplings, we find that the sign problem worsens exponentially, restricting the applicability of our approach to weak SO couplings. Since in applications, SO effects are usually weak, however, this restriction is not too severe.

In order to eliminate the spin contamination problem in the limit of zero SO coupling, we may study a few finite but small values for the SO couplings, and then extrapolate $\alpha_{R / D} \rightarrow 0$. This allows to reliably compute, for instance, the addition spectrum of the dot, where we find peaks (corresponding to stability islands of these Wigner molecules) for $N=3$ and $N=7$ electrons. Furthermore, we compute the dependence of the spin state, $\left\langle\mathbf{S}^{2}\right\rangle=S(S+1)$, as a function of particle number $N$, where $\mathbf{S}$ is the total spin operator.

The structure of the paper is as follows. After introducing the model in Section II we derive the short-time propagator for interacting fermions in a parabolic QD subject to either Rashba or Dresselhaus SO coupling (or both of them at the same time), and discuss the numerical scheme in some detail. Numerical results are presented in Section III and we conclude in Section IV] Throughout the paper, we put $\hbar=1$.

\section{MODEL AND METHOD}

We study the $N$-electron hamiltonian describing a closed parabolic dot in a 2D electron gas,

$$
H=\sum_{i=1}^{N}\left(\frac{\mathbf{p}_{i}^{2}}{2 m^{*}}+\frac{m^{*} \omega_{0}^{2}}{2} \mathbf{r}_{i}^{2}\right)+\sum_{i<j} \frac{e^{2} / \kappa}{\left|\mathbf{r}_{i}-\mathbf{r}_{j}\right|}+\sum_{i=1}^{N} H_{S O}^{(i)}
$$

where $m^{*}$ is the effective electron mass and $\omega_{0}$ is the oscillator frequency. With $i=1, \ldots, N$, the vectors $\mathbf{p}_{i}, \mathbf{r}_{i}$ denote the 2D momenta and space coordinates of all $N$ electrons. The Coulomb potential among the electrons contains screening effects of the host material via the dielectric constant, $\kappa$. Measuring energies (lengths) in units of $\omega_{0}\left(l_{0}=1 / \sqrt{m^{*} \omega_{0}}\right)$, a dimensionless Coulomb interaction parameter is given by $\lambda=e^{2} /\left(\kappa l_{0} \omega_{0}\right)$. For common host materials, $l_{0}$ is in the range of few up to hundreds of $n m$. The confinement energy $\hbar \omega_{0}$ is then typically between 0.1 up to a few $m e V$, which allows directly to determine the strength of the Coulomb interaction $\lambda$. Typical $\lambda$ currently realized experimentally are between 0.5 and 5 , the latter value [4] being already quite close to the regime studied in our paper. For $\lambda>1$, Coulomb repulsion starts to dominate over the kinetic energy, and electrons spatially arrange on shells. This "Wigner molecule" regime [18] is studied in this work.

Let us then address the SO couplings considered here. Typically, two different SO couplings are of paramount importance in semiconductor heterostructures, namely the (linear) Dresselhaus and the Rashba SO coupling. We allow for both types and consider the spin-orbit hamiltonian (of the $i$ th particle)

$$
H_{S O}=\alpha_{R}\left(p_{x} \sigma^{y}-p_{y} \sigma^{x}\right)+\alpha_{D}\left(p_{x} \sigma^{x}-p_{y} \sigma^{y}\right)=\mathbf{p} \cdot A \cdot \vec{\sigma},
$$

where the standard Pauli matrices $\vec{\sigma}=\left(\sigma^{x}, \sigma^{y}\right)$ act in spin space and

$$
A=\left(\begin{array}{cc}
\alpha_{D} & \alpha_{R} \\
-\alpha_{R} & -\alpha_{D}
\end{array}\right)
$$

Note that both types of SO coupling can be transformed into each other by a unitary transformation. Hence the spectra for $\alpha_{D}=0\left(\alpha_{R} \neq 0\right)$ and $\alpha_{R}=0\left(\alpha_{D} \neq 0\right)$ coincide (for zero magnetic field), see also Sec. III below.

Remarkably, even on the single-particle level, there is no closed solution to the Schrödinger equation in a parabolic potential subject to SO coupling of any kind. We mention in passing that for a cylindrical box, the single-particle problem has been solved analytically in Ref. 30]. PIMC simulations provide a powerful tool to 
extract numerically exact results for this few-body interacting quantum system. One starts by discretizing imaginary time $\left(0 \leq t<\beta=1 / k_{B} T\right)$ into sufficiently short time intervals $\tau=\beta / P$, where $P$ is the (integer) Trotter number. For $t=\tau$, an approximate but accurate short-time propagator can be constructed via the Trotter-Suzuki decomposition. To that end, we split $H$ into the noninteracting part $H_{0}$ (including the $\mathrm{SO}$ couplings) and the remaining interaction part $(\sim \lambda)$. Assuming that the spin dynamics is slow on the timescale $\tau$, some algebra along the lines of Ref. 38] then gives the short-time single-particle propagator under $H_{0}$ in the form

$$
\begin{gathered}
\left\langle\mathbf{r}_{2}, \sigma_{2}\left|e^{-\tau H_{0}}\right| \mathbf{r}_{1}, \sigma_{1}\right\rangle=\frac{e^{\tau E_{0}}}{2 \pi l_{0}^{2} \sinh \left(\omega_{0} \tau\right)} \\
\times e^{-S_{0}\left[\mathbf{r}_{2}, \mathbf{r}_{1}\right]}\left\langle\sigma_{2}\left|e^{-i m^{*}\left(\mathbf{r}_{2}-\mathbf{r}_{1}\right) \cdot A \vec{\sigma}}\right| \sigma_{1}\right\rangle
\end{gathered}
$$

where $E_{0}=m^{*}\left(\alpha_{R}^{2}+\alpha_{D}^{2}\right)$. The electron is described by its position $\mathbf{r}$ and the $z$-component $\sigma / 2$ of the spin. Furthermore, $S_{0}$ denotes the standard $2 \mathrm{D}$ oscillator action

$$
S_{0}\left[\mathbf{r}_{2}, \mathbf{r}_{1}\right]=\frac{\left(\mathbf{r}_{1}^{2}+\mathbf{r}_{2}^{2}\right) \cosh \left(\omega_{0} \tau\right)-2 \mathbf{r}_{1} \cdot \mathbf{r}_{2}}{2 l_{0}^{2} \sinh \left(\omega_{0} \tau\right)} .
$$

Note that in the absence of SO couplings, the exact propagator of the harmonic oscillator is reproduced. For small coupling constants $\alpha_{D, R}$ and sufficiently short $\tau$, Eq. (3) represents a very accurate short-time approximation to the single-particle propagator. The spin part in the propagator (3) can be written as

$$
\begin{aligned}
& \left\langle\sigma_{2}\left|e^{-i m^{*}\left(\mathbf{r}_{2}-\mathbf{r}_{1}\right) \cdot A \vec{\sigma}}\right| \sigma_{1}\right\rangle=\cos (a) \delta_{\sigma_{1}, \sigma_{2}} \\
& -i \frac{\sin (a)}{a}\left(a_{x}+i \sigma_{1} a_{y}\right) \delta_{\sigma_{1},-\sigma_{2}},
\end{aligned}
$$

where $\mathbf{a}=\left(a_{x}, a_{y}\right)=m^{*} A^{T}\left(\mathbf{r}_{2}-\mathbf{r}_{1}\right)$ and $a=|\mathbf{a}|$. Using Eq. (3), the many-body propagator follows in the form of $N \times N$ Slater determinants. Let us then denote the coordinate and the spin $\sigma$ of the $i$ th electron on time slice $n$ (where $1 \leq n \leq P$ ) as $\mathbf{r}_{i n}$ and $\sigma_{i n}$, respectively. With respect to the time direction, we have periodic boundary conditions. Including Coulomb interactions, we then obtain the many-particle partition function for given discretization $\tau=\beta / P$ in the form

$$
\begin{aligned}
Z & =\sum_{\left\{\sigma_{j n}= \pm\right\}} \int \prod_{n, j} d \mathbf{r}_{j n}\left(\prod_{n=1}^{P} \operatorname{det}\left(M^{(n)}\right)\right) \\
& \times \exp \left(-\sum_{n, i<j} \frac{\tau \lambda}{\left|\mathbf{r}_{i, n}-\mathbf{r}_{j, n}\right|}\right),
\end{aligned}
$$

where the $N \times N$ matrix $M^{(n)}$ has the matrix elements

$$
M_{i j}^{(n)}=\left\langle\mathbf{r}_{i, n+1}, \sigma_{i, n+1}\left|e^{-\tau H_{0}}\right| \mathbf{r}_{j, n}, \sigma_{j, n}\right\rangle .
$$

The last term in Eq. (4) represents the Coulomb interaction between all $N$ electrons confined to the QD. If there is no SO coupling, $\operatorname{det} M^{(n)}$ factorizes into a spinup and a spin-down part, $S_{z}$ is a constant of motion, and the spin contamination problem arises. We mention in passing that the weight in the discretized path integral (4) is complex-valued, and one therefore may expect that observables have an imaginary part. However, all statistical averages for physical observables must have zero imaginary part, and this indeed we find within the standard stochastic error bars. The discretized canonical many-particle partition function (44) then allows us to access equilibrium observables of interest. For concreteness, we have chosen a rather low but finite temperature, $k_{B} T / \omega_{0}=0.1$. Furthermore, unless stated otherwise, simulations were carried out for $\alpha_{D}=0$ and interaction strength $\lambda=10$, which puts us into the Wigner molecule regime. Note that by simply replacing $\alpha_{R} \rightarrow \alpha_{D}$, results for $\alpha_{R}=0$ follow.

The main limitation for this type of PIMC simulation comes from the fermionic sign problem. The sign problem generally arises when different paths that contribute to averages carry different signs, or even complexvalued phases, as encountered in the case of non-zero SO couplings. The resulting sign cancellation when sampling fermion paths then manifests itself as a very small signal-to-noise ratio. For instance, as a consequence of exchange, these phases appear when forming Slater determinants. Here, in the presence of SO couplings, the sign problem occurs even for a single particle. Unfortunately, as a function of SO couplings, we find an exponential decay of the sign, see Fig. 1 This can be rationalized already on the single-particle level, since the propagator acquires a complex phase factor in the presence of $\mathrm{SO}$ couplings. The propagator then resembles a real-time propagator for a single particle, with time corresponding to the SO coupling. For this problem, the exponential severity of the sign problem is well established. In effect, the parameter regime where reliable simulations are possible is limited to small-to-intermediate $\mathrm{SO}$ couplings. We note in passing that the Rashba SO coupling $\alpha_{R}=(0.4-1.1) \times 10^{-11} \mathrm{eVm}$ reported for the InGaAs dots of Ref. [8] are about one order of magnitude larger than our largest value. However, the SO coupling in InGaAs is also unusally strong, and our values should apply more directly to GaAs dots. Simultaneously, the sign problem becomes more severe when increasing $N$ or lowering temperature. For the chosen parameters, the sign problem only allows to study QDs containing $N<10$ electrons. The average sign is $\langle\phi\rangle>0.001$ in all cases reported below.

We then compute several observables. First, the energy of the $N$-electron dot can be obtained from

$$
E_{N}=-\frac{\partial \ln Z(\beta)}{\partial \beta},
$$

where the derivative can be explicitly carried out using Eq. (4). Knowledge of the $E_{N}$ determines the addition energy

$$
\Delta(N)=E_{N+1}-2 E_{N}+E_{N-1} .
$$


A peak in the addition energy $\Delta(N)$ indicates enhanced stability of the $N$-electron dot ("magic number") [1]. Note that experimentally observed addition energies are determined by free energy differences, while we compute the energy. However, for the low-temperature regime studied here, entropic contributions are smaller by about one order of magnitude, and therefore our values for $\Delta(N)$ are of relevance to actual experiments. Another quantity of interest is the total spin $S$, which we extract from the definition

$$
\left\langle\mathbf{S}^{2}\right\rangle=S(S+1),
$$

where brackets denote the statistical average using Eq. (4), and $\mathbf{S}$ is the total spin operator. Finally, spatial ordering can be monitored via the electronic particle density,

$$
n(\mathbf{r})=\sum_{i=1}^{N}\left\langle\delta\left(\mathbf{r}-\mathbf{r}_{i}\right)\right\rangle,
$$

which is normalized to $\int d \mathbf{r} n(\mathbf{r})=N$. The related charge densities can also be accessed experimentally, e.g., via capacitance spectroscopy or scanning tunneling microscopy techniques [4].

As we work with finite discretization $\tau$, Trotter approximation errors have to be taken into account [39]. As shown in Ref. [40], for small $\tau$, such errors vanish quadratically for all observables, allowing for simple and efficient extrapolation schemes that completely eliminate this finite- $\tau$ error. The $\tau^{2}$ scaling regime is reached for $\tau \omega_{0} \leq 0.35$ when computing the energy. Furthermore, particle or spin densities are found to reach the $\tau^{2}$ scaling regime already at higher $\tau$. Results shown here have been carefully extrapolated down to $\tau=0$ with a linear regression fit, using results from several simulations obtained at $1 / 6 \leq \tau \omega_{0} \leq 1 / 3$. Hence no discretization errors are present, see also Ref. [21], and error bars denote just the standard stochastic Monte Carlo errors.

Spin flip moves are an essential ingredient of the algorithm. Within the PIMC we allow for spin flips as well as for position moves. Single-particle moves were found sufficient to ensure ergodicity. The average trial step size for position moves was adjusted to give acceptance rates of the order of $30 \%$. On the other hand, typical spin-flip acceptance rates were much lower (several percent) and strongly dependent on $\alpha_{R / D}$. The possibility to change $S_{z}$ arises from the SO coupling and can be used to circumvent the spin contamination problem even for the case of no SO coupling, namely by extrapolating finite$\alpha_{R}$ results (where spins can be flipped and no spin contamination problem is present) down to $\alpha_{R} \rightarrow 0$. Such a scheme yields the energy $E_{N}$ as well as the expectation value $\left\langle\mathbf{S}^{2}\right\rangle$ for the total spin of the many-body system at finite temperature. Typically, after $\approx 10^{3}$ equilibration passes, $1.5 \times 10^{7} \mathrm{MC}$ samples were accumulated for each parameter set. Our code runs at a speed of up to two weeks (for $N=9, P=60$ ) for a given parameter set per $1.5 \times 10^{7}$ samples on a standard $2 \mathrm{GHz}$ Xeon processor.
We have checked our PIMC energies for $N=2$ against finite-temperature exact diagonalization results, including both interactions and spin-orbit couplings. We found excellent agreement, validating our approach.

\section{RESULTS}

To verify that for $\lambda=10$, we indeed have a Wigner molecule [18], let us start with the radially integrated particle density (8) shown in Fig. 2] The cylindrical symmetry of the QD implies that $n(\mathbf{r})$ only depends on the modulus $r=\mathbf{r}$. The plot indicates that the sixth electron enters the center of the dot, whereas the remaining five electrons arrange on an outer ring in order to minimize the Coulomb repulsion. More electrons are then added to the outermost shell. Finally, for $N=9$, a second electron enters the center. This spatial shell filling sequence (as opposed to orbital shell filling) is typical of the Wigner molecule, which forms the finite-size counterpart of a Wigner crystal. In fact, precisely this spatial filling sequence has been reported from a purely classical analysis (in particular, disregarding spin effects) [25, 26]. At higher temperatures, the Wigner molecule melts via thermal fluctuations, while for lower $\lambda$, it is eventually destroyed by quantum fluctuations. Generally, we find that particle densities are practically independent of the $\mathrm{SO}$ coupling strengths $\alpha_{R}$ or $\alpha_{D}$, at least for $\alpha_{R / D} l_{0} \leq 0.05$.

Although we consider rather small SO couplings, the many-body energy $E_{N}$ can be clearly seen to decrease as a function of $\alpha_{R}$ (here, $\alpha_{D}=0$ ). This trend has been observed in other studies as well 34. In Fig. B we show this effect for $N=3$ and $N=4$ electrons.

For $\alpha_{R} l_{0} \leq 0.07$, the $\mathrm{SO}$ coupling does not significantly influence addition energies for $N \leq 5$, see Fig. 目 However, there is a slight increase in the addition energy $\Delta(6)$, while the peak for $N=7$ is reduced for $\alpha_{R}=0.04$ (dotted curve). (In particular, $\Delta(7)=3.00(1)$ for $\alpha_{R} \rightarrow 0$, while $\Delta(7)=2.95(2)$ at $\alpha_{R}=0.04$.) Remarkably, the magic numbers $N=3$ and $N=7$ encountered in the Wigner molecule regime are different from the ones for weak interactions (where a standard Fermi liquid phase is present). This is indicated in the

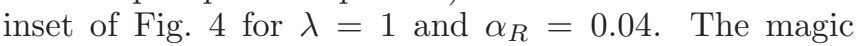
numbers for $\lambda=1$ can be rationalized in terms of the subsequent filling of energy levels (orbitals) of a 2D harmonic oscillator. This predicts a peak for $N=2$, where the lowest level is filled, and another peak at $N=4$ reflecting Hund's rule behavior, see also Ref. 35]. For strong Coulomb repulsion, the peak for $N=2$ is completely absent, while $N=3$ now corresponds to a magic number. A very distinct peak in the addition energy is observed for $N=7$. To the best of our knowledge, this peak is not expected for weak interactions. The picture of filling up spatial shells in the Wigner solid phase (discussed above) may suggest that the filled spatial shell configurations $N=5$ and $N=8$ represent magic numbers. However, under a classical reasoning, the addition 
energy should have no pronounced peaks but exhibits a rather smooth and monotonic decay in $\Delta(N)$ 25, 26]. Moreover, the classical prediction for $\Delta(N)$ 26] yields values one order of magnitude smaller than the $\Delta(N)$ found here. This indicates that for $\lambda=10$, despite the clear onset of spatial ordering, quantum effects are still very important and cause the magic numbers $N=3$ and $N=7$. Notably, for these $N$, the dot is seen to be partially spin-polarized. The magic numbers for $N=3$ and $N=7$ can thus be rationalized in terms of a Hund-rule type behavior specific to the incipient Wigner crystallized regime (see the discussion below and Fig. 6). Our findings are therefore characteristic for the quantum character of the Wigner molecule. A purely classical "Wigner solid" analysis [25, 26] is expected to apply only for extremely large $\lambda$. Our numerical PIMC results for $E_{N}$ (and the spin) as a function of $N$ and the Rashba coupling $\alpha_{R}$ are summarized in Table【 These data were all obtained for $\lambda=10$ and $k_{B} T / \omega_{0}=0.1$

Next we consider the dependence of the energy on the two types of spin couplings. Let us take $N=4$ electrons, again at $\lambda=10$, and fix $\left(\alpha_{R}+\alpha_{D}\right) l_{0}=0.05$. We then study $E_{4}$ as a function of $\gamma=\left(\alpha_{R}-\alpha_{D}\right) /\left(\alpha_{R}+\alpha_{D}\right)$, where $-1 \leq \gamma \leq 1$ tunes the relative strength of Rashba versus Dresselhaus coupling. The result is depicted in Fig. 5] showing a symmetric curve $\delta E_{4}(\gamma)=E_{(0,0)}-$ $E_{\left(\alpha_{R}, \alpha_{D}\right)}$. The symmetric shape can be explained by the unitary spin rotation which transforms the Rashba- into the Dresselhaus term and vice versa. With increasing $|\gamma|$, the relative weights of the Dresselhaus and Rashba couplings increase, leading to increasing energy gains $\delta E_{4}$ over the system without SO couplings.

Finally, we observe that spin plays a major role for $\lambda=10$. Our data for $S$ as defined in Eq. (7) show a drastic influence of temperature. For such a strongly interacting system, energies for different $S$ are typically close by, and a thermal average can then result in different $S$ as compared to ground-state results. For instance, for $N=2$, we find a mixture of the singlet ground state $S=0$ and the excited triplet state $S=2$, as PIMC gives $S(S+1)=1.14(1)$ at $k_{B} T / \omega_{0}=0.1$. Due to the strong Coulomb interaction, there is a clear trend towards partial spin polarization as a function of $\lambda$, indicated in Fig. 6] see also Table凹 Moreover, the thermal average for $\left\langle\mathbf{S}^{2}\right\rangle$ obtained from PIMC simulations at $\lambda=10$ increases monotonically with $N$, while the ground-state spin [18] has rather different values and shows a nonmonotonic dependence on $N$. It is worth mentioning that the peaks in the addition energy for $N=3$ and $N=7$ correspond to enhanced values of $S$ as well. Stability of the $N$ electron dot is thus connected to a tendency towards spin polarization, reminiscent of Hund's rule behavior.
For $\lambda=4$, corresponding to weaker but still sizeable interactions, the dependence of the ground-state spin [18] is given in the inset. The shown nonmonotonic dependence on $N$ reflects the standard Hund's rule physics. Comparing the $\lambda=4$ and $\lambda=10$ results, we observe that interactions tend to further spin-polarize the dot. The huge thermal effects observed in the expectation value $\left\langle\mathbf{S}^{2}\right\rangle$ also indicate that unless experiments are carried out at extremely low temperatures, spin blockade phenomena 41] relying on total spin selection rules will be thermally washed out. Finally, we note that for the SO couplings studied here, spin expectation values were not significantly affected.

\section{CONCLUSION}

We have investigated the behavior of up to nine electrons in a quantum dot. We took into account strong Coulomb correlations between the electrons, and also incorporated spin-orbit couplings. Our results were obtained from path-integral quantum Monte Carlo simulations. An exponential decrease of the fermionic sign is found with increasing SO couplings. This sign problem appears even for a single electron. Nevertheless, simulations are possible for weak SO couplings, where their inclusion can also be used to eliminate the spin contamination problem.

We observe peaks in the addition energy spectrum for $N=3$ and $N=7$, which are likely to correspond to the stability of partially spin-polarized configurations induced by Coulomb interactions. These peaks are neither expected in the weak-interaction regime nor in the classical (deep) Wigner solid, where spin effects are negligible. We hope that this prediction can soon be tested experimentally. Our results were obtained in the regime of weak spin-orbit couplings, since otherwise numerical instabilities associated with the sign problem occur. Given this restriction however, PIMC offers a powerful tool to analyze the effects of spin-orbit couplings in strongly interacting quantum dots. We find no dramatic effects, but observable downward shifts in the many-body energy that scale quadratically in the spin-orbit couplings. Spin-orbit couplings also affect addition energies.

\section{Acknowledgments}

This work was supported by the DFG under the Gerhard-Hess program. We thank M. Thorwart for a careful reading of the manuscript.
[1] S.M. Reimann and M. Manninen, Rev. Mod. Phys. 74, 1283 (2002), and references therein.

[2] Y. Alhassid, Rev. Mod. Phys. 72, 895 (2000).
[3] L.P. Kouwenhoven, D.G. Austing, and S. Tarucha, Rep. Prog. Phys 64, 701 (2001).

[4] R.C. Ashoori, Nature 379, 413 (1996). 
[5] S. Tarucha, D.G. Austing, Y. Tokura, W.G. van der Wiel, and L.P. Kouwenhoven, Phys. Rev. Lett. 84, 2485 (2000).

[6] P. Matagne, J.P. Leburton, D.G. Austing, and S. Tarucha, Phys. Rev. B 65, 085325 (2002).

[7] S. Lindemann, T. Ihn, T. Heinzel, W. Zwerger, K. Ensslin, K. Maranowski, and A.C. Gossard, Phys. Rev. B 66, 195314 (2002).

[8] J. Nitta, T. Akazaki, H. Takayanagi, and T. Enoki, Phys. Rev. Lett 78, 1335 (1997).

[9] Th. Schäpers, G. Engel, J. Lange, Th. Klocke, M. Hollfelder, and H. Lüth, J. Appl. Phys. 83, 4324 (1998).

[10] D. Grundler, Phys. Rev. Lett. 84, 6074 (2000).

[11] T. Koga, J. Nitta, T. Akazaki, and H. Takayanagi, Phys. Rev. Lett. 89, 046801 (2002).

[12] J. Könemann, R.J. Haug, D.K. Maude, V.I. Fal'ko, and B.L. Altshuler, Phys. Rev. Lett. 94, 226404 (2005).

[13] D. Loss and D.P. DiVincenzo, Phys. Rev. A 57, 120 (1998).

[14] I. Zutic, J. Fabian, and S. Das Sarma, Rev. Mod. Phys. 76, 323 (2004).

[15] E.I. Rashba, Sov. Phys. Sol. State 2, 1109 (1960); Y.A. Bychkov and E.I. Rashba, J. Phys. C 17, 6039 (1984).

[16] G. Dresselhaus, Phys. Rev. 100, 580 (1955).

[17] C.F. Destefani and S.E. Ulloa, Phys. Rev. B 71, 161303(R) (2005).

[18] R. Egger, W. Häusler, C.H. Mak, and H. Grabert, Phys. Rev. Lett. 82, 3320 (1999); ibid. 83, 462(E) (1999).

[19] C.H. Mak, R. Egger, and H. Weber-Gottschick, Phys. Rev. Lett. 81, 4533 (1998).

[20] A.V. Filinov, M. Bonitz, and Yu.E. Lozovik, Phys. Rev. Lett. 86, 3851 (2001).

[21] B. Reusch and R. Egger, Europhys. Lett. 64, 84 (2003).

[22] W. Häusler, Europhys. Lett. 49, 231 (2000).

[23] J. Harting, O. Mülken, and P. Borrmann, Phys. Rev. B 62, 10207 (2000).

[24] M. Rontani, C. Cavazzoni, D. Bellucci, and G. Goldoni cond-mat/0508111

[25] Yu.E. Lozovik, Usp. Fiz. Nauk 153356 (1987) [Sov. Phys. Usp. 30, 912 (1987)]; Yu.E. Lozovik and L.M. Pomirchy, Phys. Status Solidi B 161, K11 (1990).

[26] F.M. Bolton and U. Rössler, Superlatt. Microstruct. 13, 139 (1993); V.M. Bedanov and F.M. Peeters, Phys. Rev. B 49, 2667 (1994).

[27] S.A. Mikhailov, Phys. Rev. B 65, 115312 (2002).

[28] F. Pederiva, C.J. Umrigar, and E. Lipparini, Phys. Rev. B 62, 8120 (2000).

[29] A. Harju, S. Siljamäki, and R.M. Nieminen, Phys. Rev. B 65, 075309 (2002).

[30] E. Tsitsishvili, G.S. Lozano, and A.O. Gogolin, Phys. Rev. B 70, 115316 (2004).

[31] O. Voskoboynikov, C.P. Lee, and O. Tretyak, Phys. Rev. B 63, 165306 (2001).

[32] W.H. Kuang, C.S. Tang, and W. Xu, J. Appl. Phys. 95 6368 (2004).

[33] T. Chakraborty and P. Pietiläinen, Phys. Rev. B 71, 113305 (2005).

[34] C.F. Destefani, S.E. Ulloa, and G.E. Marques, Phys. Rev. B 69, 125302 (2004); Phys. Rev. B 70, 205315 (2004).

[35] M. Governale, Phys. Rev. Lett. 89, 206802 (2002).

[36] A. Emperador, E. Lipparini, and F. Pederiva, Phys. Rev. B 70, 125302 (2004).

[37] A. Ghosal, C.J. Umrigar, H. Jiang, D. Ullmo, and H.U. Baranger, Phys. Rev. B 71, 241306(R) (2005).

[38] J. Zinn-Justin, Quantum Field Theory and Critical Phe- nomena, 4th edition, Chapter 3 (Clarendon Press, Oxford, 2002).

[39] H. De Raedt and B. De Raedt, Phys. Rev. A 28, 3575 (1983).

[40] R.M. Fye, Phys. Rev B 33, 6271 (1986).

[41] D. Weinmann, W. Häusler, and B. Kramer, Phys. Rev. Lett. 74, 984 (1995). 


\begin{tabular}{|ll|l|l|}
\hline$N$ & $\alpha_{R} l_{0}$ & $E / \omega_{0}$ & $S(S+1)$ \\
\hline \hline 1 & 0 & $0.9988(1)$ & $0.75(0)$ \\
1 & 0.04 & $0.9986(6)$ & $0.75(0)$ \\
\hline 2 & 0 & $7.464(2)$ & $1.14(1)$ \\
2 & 0.04 & $7.459(3)$ & $1.11(2)$ \\
\hline 3 & 0 & $17.610(1)$ & $2.424(13)$ \\
3 & 0.04 & $17.603(2)$ & $2.426(16)$ \\
\hline 4 & 0 & $31.454(1)$ & $2.657(4)$ \\
4 & 0.04 & $31.448(6)$ & $2.654(31)$ \\
\hline 5 & 0 & $48.717(1)$ & $3.312(26)$ \\
5 & 0.04 & $48.712(14)$ & $3.339(71)$ \\
\hline 6 & 0 & $68.959(1)$ & $4.280(13)$ \\
6 & 0.04 & $68.917(30)$ & $4.27(12)$ \\
\hline 7 & 0 & 91.929 & $4.96(18)$ \\
7 & 0.04 & $91.906(30)$ & $4.89(11)$ \\
\hline 8 & 0 & 117.889 & $5.307(89)$ \\
8 & 0.04 & $117.83(6)$ & $5.37(35)$ \\
\hline 9 & 0 & $146.501(1)$ & $5.67(19)$ \\
9 & 0.04 & $146.36(22)$ & $5.95(68)$ \\
\hline
\end{tabular}

TABLE I: PIMC data for $E_{N}$ and $S(S+1)=\left\langle\mathbf{S}^{2}\right\rangle$ as a function of $\alpha_{R}$ for $\alpha_{D}=0, \lambda=10$, and $k_{B} T / \omega_{0}=0.1$. The $\alpha_{R}=0$ data are taken from extrapolations. Bracketed numbers denote error estimates.

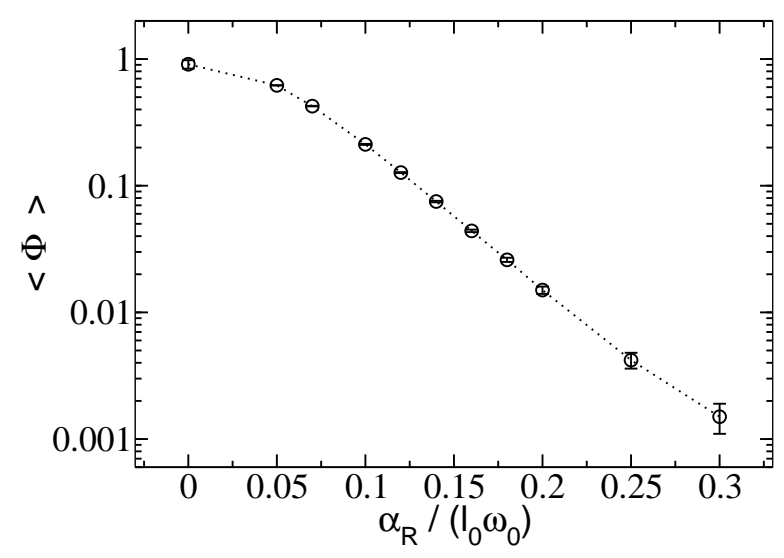

FIG. 1: Average sign $\langle\phi\rangle$ as a function of $\alpha_{R}$ (semilogarithmic scale), for $N=3, \alpha_{D}=0$ and $\tau \omega_{0}=0.25$. The dotted curve is a guide to the eye only. Vertical bars denote standard Monte Carlo error bars (one standard deviation).

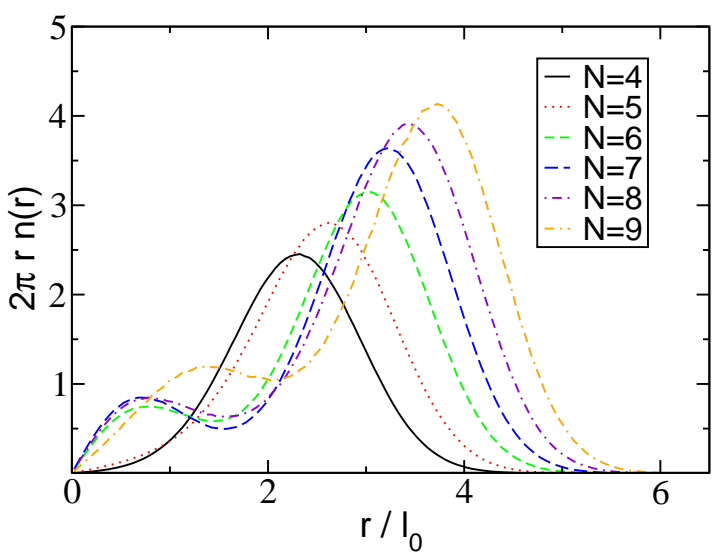

FIG. 2: (Color online) Charge density for $N=4$ to $N=9$ electrons at $\alpha_{R} l_{0}=0.02$ and $\alpha_{D}=0, \lambda=10$ and $k_{B} T / \omega_{0}=$ 0.1 . 


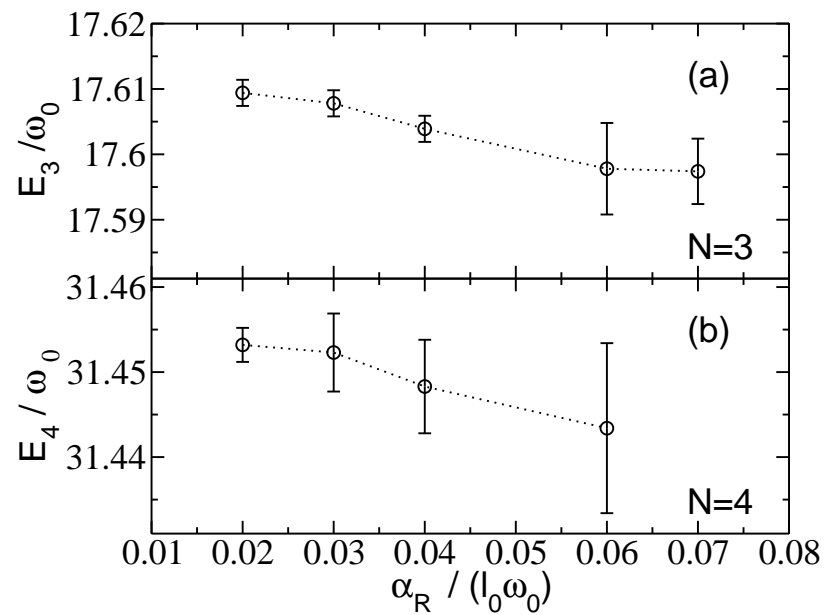

FIG. 3: Energy $E_{N}$ in units of $\omega_{0}$ for $(a)$ three and $(b)$ four electrons in the QD as a function of $\alpha_{R}$ (here $\left.\alpha_{D}=0\right)$. With increasing $\alpha_{R}$, the sign problem becomes more severe, and thus the $\mathrm{MC}$ error bars tend to increase.

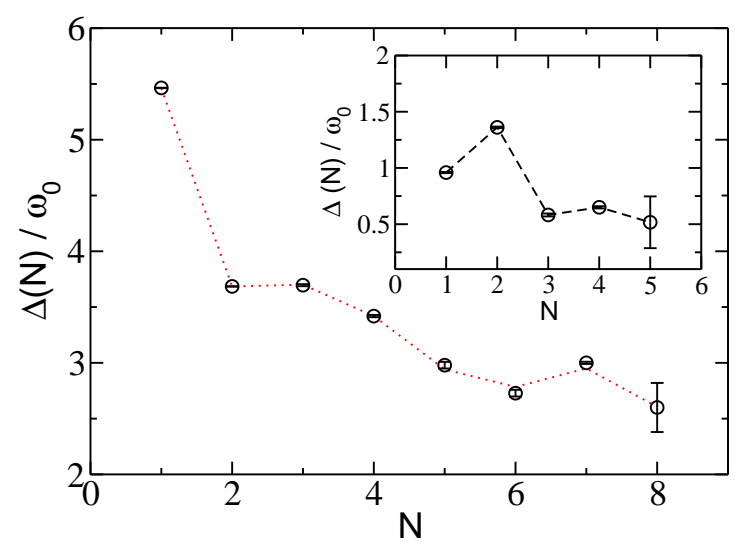

FIG. 4: (Color online) Addition energy $\Delta(N)$ in units of $\omega_{0}$ for $\lambda=10$. Circles denote PIMC data extrapolated to $\alpha_{R}=0$. Note that $N=3$ represents a peak, since $\Delta(3)=$ $3.696(9)$, whereas $\Delta(2)=3.685$ and $\Delta(4)=3.419(10)$. The dotted curve connects the corresponding PIMC results for $\alpha_{R}=0.04$. Inset: Same for $\lambda=1$ and small $N$. The dashed curve is a guide to the eye only. 


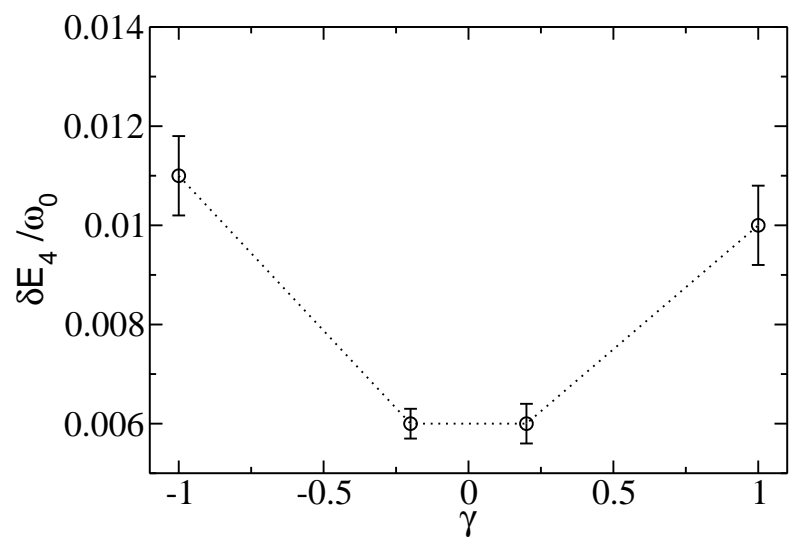

FIG. 5: Energy difference $\delta E_{4}=E_{(0,0)}-E_{\left(\alpha_{R}, \alpha_{D}\right)}$ for $N=4$ and several $\gamma$. The dotted line is a guide to the eye only.

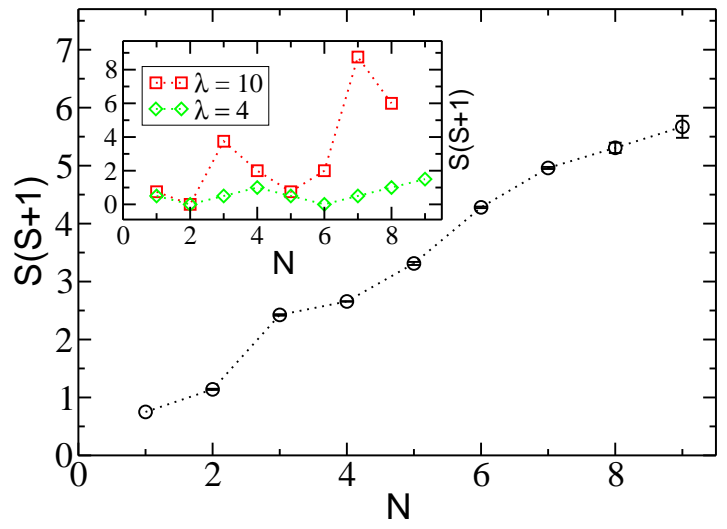

FIG. 6: (Color online) $S(S+1)$ as defined in Eq. (7) as a function of $N$ for $\lambda=10$ and $k_{B} T / \omega_{0}=0.1$. Dotted or dashed lines are guides to the eye only. Inset: Ground-state $\left(T=0, \alpha_{R / D}=0\right)$ value for $S(S+1)$ from Ref. [18] for $\lambda=10$ (squares) and for $\lambda=4$ (diamonds). 